\title{
FLAVIN MONONUCLEOTIDE-EXFOLIATED GRAPHENE FLAKES AS ELECTRODES FOR THE ELECTROCHEMICAL DETERMINATION OF URIC ACID IN THE PRESENCE OF ASCORBIC ACID
}

\author{
A. Abellán-Llobregat ${ }^{1}$, M. Ayán-Varela ${ }^{2}$, L. Vidal ${ }^{3}$, J.I. Paredes ${ }^{2}$, S. Villar-Rodil ${ }^{2}$, \\ A. Canals ${ }^{3}$, E. Morallón ${ }^{1}$ \\ 1 Instituto Universitario de Materiales. Universidad de Alicante. Apartado 99. 03080 \\ Alicante. Spain. \\ ${ }^{2}$ Instituto Nacional del Carbón, INCAR-CSIC, Apartado 73, 33080 Oviedo, Spain. \\ ${ }^{3}$ Departamento de Química Analítica, Nutrición y Bromatología and Instituto Universitario \\ de Materiales. Universidad de Alicante. Apartado 99. 03080 Alicante. Spain.
}

\begin{abstract}
Gold interdigitated microelectrodes (Au-IDA) modified with either graphene flakes exfoliated using flavin mononucleotide (FMN) (Gr-FMN) or graphene flakes and platinum nanoparticles (Pt-GrFMN) have been studied in the oxidation of uric acid (UA). An electrochemical method for the detection and quantification of UA in phosphate buffer solution at physiological pH (PBS, $0.25 \mathrm{M}$, $\mathrm{pH}$ 7) in the absence and presence of ascorbic acid (AA) has been studied by cyclic voltammetry. The quantification of UA was investigated by cyclic voltammetry, presenting an oxidation peak at $0.99 \mathrm{~V}$ with both modified electrodes. Linearity range of 60-578 $\mu \mathrm{M}$ and $60-345 \mu \mathrm{M}$ has been found for Gr-FMN/Au-IDA and Pt-Gr-FMN/Au-IDA electrodes, respectively. Limits of detection of $18 \mu \mathrm{M}$ were obtained for both electrodes, and the repeatability was studied at $177 \mu \mathrm{M}$ providing 4\% and 8\% for Gr-FMN/Au-IDA and Pt-Gr-FMN/Au-IDA, respectively. AA interference has been studied by cyclic voltammetry, showing two clearly separated oxidation peaks, at $0.99 \mathrm{~V}$ for UA oxidation and at $0.74 \mathrm{~V}$ for Gr-FMN/Au-IDA and $0.70 \mathrm{~V}$ for Pt-Gr-FMN/Au-IDA for AA oxidation. Linearity range has been studied in presence of $250 \mu \mathrm{M}$ AA obtaining a working range of 60-578 $\mu \mathrm{M}$ for Gr-FMN/Au-IDA electrode and of 60-288 $\mu \mathrm{M}$ with Pt-Gr-FMN/Au-IDA electrode. Limits of detection remain at $18 \mu \mathrm{M}$ for both electrodes and the repeatability was studied at $177 \mu \mathrm{M}$ providing $8 \%$ and 14\% for Gr-FMN/Au-IDA and Pt-Gr-FMN/Au-IDA electrodes respectively.
\end{abstract}




\section{Introduction}

Uric acid (2,6,8-trihydroxypurine, UA) is the primary end product of purine metabolism. The human normal range of UA is from 240 to $520 \mu \mathrm{M}$ in serum and 1.4-4.4 $\mathrm{mM}$ in urine [1], [2], [3]. In the last years the detection and quantification of UA has assumed a major role due to the fact that high levels of this analyte are related with several diseases such gout, obesity, Lesch-Nyan disease, diabetes, high cholesterol or heart diseases [4],[5],[6],[7]. However, concentrations lower than the established minimum have been linked with Parkinson, Alzheimer or multiple sclerosis [1]. For the reasons described above, the establishment of a method to determine accurately UA levels in human physiological fluids is mandatory.

The common method employed in clinical laboratories to determine the concentration of UA in human fluids is based on the specific enzymatic oxidation of UA [2]. Other method is high performance liquid chromatography with UV absorbance detection [8]. However, the use of the electrochemical methods provides some advantages, such as short analysis time, simple experimental procedures, relatively economical instrumental requirements and high selectivity and sensitivity [2]. As a result, electrochemical techniques have been widely employed for the determination of several analytes, including UA and AA.

In spite of all the advantages described above, the most important disadvantage of the electrochemical detection and quantification of UA is its high oxidation potential that produces the interference of several compounds, obtaining an overlapped signal. Then, the development of electrode materials with an efficient capability towards separating the oxidation peaks of UA from those of other compounds in its voltammetric determination is highly demanded. In this context, carbon materials are usually employed for electroanalysis as a result of their attractive electrochemical properties such as low residual current, readily renewable surface and wide aqueous potential window [9]. Colín-Orozco et al. [10] studied the electrochemical behaviour of UA, dopamine (DP) and AA by cyclic voltammetry and differential pulse voltammetry (DPV) in a $0.1 \mathrm{M} \mathrm{NaCl}$ aqueous solution and by using a bare carbon paste electrode (CPE), achieving by DPV a suitable sensitivity $\left((23.79 \pm 0.05) \mu \mathrm{A} \cdot \mathrm{mM}^{-1}\right)$ and low limit of quantification of $(18.18 \pm 0.05) \mu \mathrm{M}$ for UA quantification. However, the electrochemical study performed by Colín-Orozco et al. was carried out in media where UA, DA and AA were not mixed [10], and the simultaneous detection of UA in the presence of AA was not studied. Actually, the direct electrochemical detection of UA in the presence of other species (e.g., xanthine and hypoxanthine) using a glassy carbon electrode has been reported by other groups [11]. However, when AA is in the sample, the electrochemical 
detection of UA is not possible, due to the adsorption of the AA oxidation product on the glassy carbon electrode surface, resulting in an unusable electrode [12]. Therefore, several modified glassy carbon electrodes [13] and the use of other carbon material have been proposed for this application [14],[15],[16].

Among carbon nanomaterials, graphene has acquired an important role as a material for electrochemical sensing [9],[17], due to its unique properties including fast electron transport, high thermal conductivity, mechanical flexibility and good biocompatibility [9]. Furthermore, singlelayer graphene has a high surface area (theoretically $2630 \mathrm{~m}^{2} \mathrm{~g}^{-1}$ ) [17], which makes it ideal as a support material, and also the presence of defects that can act as electroactive sites [18]. Several papers have been found in the literature where graphene or graphene-related materials have been employed for the development of electrochemical sensors. For instance, Shang et al. [19] use multilayer graphene nanoflake films for the simultaneous detection of UA, AA and dopamine (DP), achieving a great separation of the signals obtained by cyclic voltammetry. Sun et al. [20] have developed a UA sensor based on graphene oxide modified with thionine, obtaining a low detection limit of $7 \mu \mathrm{M}$ without any interference from AA and DP. However, in the cases describe above, the materials preparation was tedious and the use of uricase enzyme was necessary, resulting in an increase in the total cost of the sensor. Other references related with UA quantification by voltammetric detection have been found in the literature, showing linear ranges in biological fluids. However the sensitivity achieved in these works is lower than the desirable [3], [21], [22], [23]. In order to increase the sensitivity, metal nanoparticles are used. Then, platinum nanoparticles supported on a graphene material should be a suitable strategy to reduce costs while maintaining the electrocatalytic activity of platinum [24]. In addition, another drawback that needs to be solved is the use of organic solvents for the dispersion of the electrode material, especially graphene. Aqueous suspensions of high concentrations of graphene flakes for the preparation/modification of electrodes are desirable; however, due to hydrophobic properties of graphene these dispersions are difficult to prepare. Then, the dispersants should fulfil some requirements to be employed: 1) It should lead to very high concentrations of colloidal stabilized graphene sheets. 2) The amount of dispersant has to be very low and 3) it should be innocuous and nontoxic [25]. Regarding these points, Ayán-Varela et al. [25] have very recently developed a method to prepare stable aqueous graphene dispersions using the sodium salt of flavin mononucleotide (FMN), achieving extremely concentrated dispersions of high quality graphene flakes. These dispersions have been used in the preparation of supported metallic nanoparticles (Pt, Pd, Ag) [25]. 
Based on the work above, taking advantage of the attractive properties of graphene and using the method developed to prepare highly concentrated aqueous dispersions of graphene, in this work graphene-modified arrays of gold interdigitated microelectrodes are presented for UA voltammetric detection in the presence of AA. The gold interdigitated microelectrodes have been modified with FMN-stabilized graphene dispersion (Gr-FMN) and with Pt nanoparticles on FMN-stabilized graphene dispersion (Pt-Gr-FMN). To our knowledge, this is the first time that these modified electrodes have been used for this purpose.

\section{Experimental}

\subsection{Reagents and equipment}

Uric acid ( $\geq 99 \%$, crystalline) and L-Ascorbic acid (reagent grade, crystalline) standard chemicals were provided by Sigma-Aldrich. Potassium dihydrogen phosphate $\left(\mathrm{KH}_{2} \mathrm{PO}_{4}\right)$ and dipotassium hydrogen phosphate $\left(\mathrm{K}_{2} \mathrm{HPO}_{4}\right)$ obtained from Emsure ${ }^{\circledR}$ and Sigma-Aldrich, respectively, were used to prepare phosphate buffer solution (PBS, $0.25 \mathrm{M}$, pH 7). Sulphuric acid (98\%) was provided by AnalaR Normapur ${ }^{\circledR}$ and sodium hydroxide $(\mathrm{NaOH})$ from Merck. All the solutions were prepared with 18.2 $\mathrm{M} \Omega \mathrm{cm}$ ultrapure water obtained from an Elga Labwater Purelab system.

Graphene flakes exfoliated and stabilized with flavin mononucleotide (Gr-FMN) and Gr-FMN hybridized with Pt nanoparticles (Pt-Gr-FMN) were prepared according to the procedure described in the literature [25].

Transmission electron microscopy (TEM) imaging was carried out using a JEOL TEM, JEM-2010 microscope, which was furnished with an Oxford X-ray detector (EDS) (INCA Energy TEM 100 model) and GATAN acquisition camera (ORIUS SC600).

X-ray photoelectron spectroscopy (XPS) was performed using a VG-Microtech Mutilab 3000 model.

\subsection{Preparation of graphene modified electrodes}

Thin-film interdigitated array microelectrodes (IDA) of gold (Micrux Technologies) have been used as support electrodes. The following procedure has been implemented for the preparation of the graphene-modified electrodes. Firstly, the gold interdigitated array electrodes (Au-IDA) was cleaned by cyclic voltammetry performing ten cycles between $-1.5 \mathrm{~V}$ and $1.5 \mathrm{~V}$ vs gold pseudoreference at $0.1 \mathrm{~V} \cdot \mathrm{s}^{1}$ in $0.05 \mathrm{M} \mathrm{H}_{2} \mathrm{SO}_{4}$. Then, the Au-IDA was modified by drop casting a given graphene dispersion. The concentration of the dispersion was $0.188 \mathrm{mg} \cdot \mathrm{mL}^{-1}$ and $0.116 \mathrm{mg} \cdot \mathrm{mL}^{-1}$ for graphene (Gr-FMN) and Pt-decorated graphene (Pt-Gr-FMN) dispersions, respectively. The 
weight of the deposited graphene or Pt-graphene hybrid was $2.35 \mu \mathrm{g}$ for all the electrodes. The method for the deposition was the addition of a defined number of $0.5 \mu \mathrm{L}$ drops (25 and 40 drops for Gr-FMN and Pt-Gr-FMN dispersions, respectively), which were allowed to dry with the use of an infrared lamp. Two different electrodes were prepared, one of them modified with Gr-FMN (GrFMN/Au-IDA) and the other one modified with Pt-Gr-FMN (Pt-Gr-FMN/Au-IDA). Finally, the electrodes were stored in PBS at room temperature until their use.

\subsection{Electrochemical methods}

All electrochemical measurements were carried out with a cyclic voltammetry sep-up (EDAQ EA 163 Model potentiostat, EG\&G PARC model 175 Universal Programmer and e-corder EDAQ 410). A three-electrode configuration has been used, with the modified electrodes (Gr-FMN/Au-IDA and Pt-Gr-FMN/Au-IDA) acting as the working electrodes, a reversible hydrogen electrode (RHE) immersed in the same electrolyte as a reference electrode and a gold wire as a counter electrode. The electrochemical cell, was completely deoxygenated during the measurements by nitrogen bubbling.

The calibration curves have been obtained by adding several aliquots from a concentrated stock solution of 100 mM UA in PBS with a pH higher than 8.4 until reaching the desired concentration, between 60 and $578 \mu \mathrm{M}$. When the interference of AA was studied, a fixed AA concentration of $250 \mu \mathrm{M}$ was added to the electrochemical cell and the same UA concentration range has been studied.

All measurements were carried out in PBS (37 mL, $0.25 \mathrm{M}, \mathrm{pH}$ 7) and by triplicate in order to determine analytical parameters. For all replicates, one modified electrode was used, and a new AA and UA solutions were prepared for each replicate.

\section{Results and Discussion}

\subsection{Modified electrode characterization}

The electrochemical behavior of the modified electrodes was investigated in the electrolyte in absence of UA by cyclic voltammetry. Figure 1 shows the voltammograms for Gr-FMN/Au-IDA and Pt-Gr-FMN/Au-IDA electrodes in PBS. For Gr-FMN/Au-IDA (Figure 1A), the voltammogram exhibits different redox processes; two overlapped at around $0.07 \mathrm{~V}$ and $0.17 \mathrm{~V}$ and a third one at 
$0.3 \mathrm{~V}$ during the positive going sweep. During the reverse scan, three reduction peaks are clearly observed.

It is widely accepted that, in aqueous media and when the riboflavin is present in solution, fully oxidized flavin $(F M N)$ is reduced to flavin-hydroquinone $\left(\mathrm{FMNH}_{2}\right)$ in "one-step" in a $2 \mathrm{e}^{-} / 2 \mathrm{H}^{+}$ reduction (scheme 1) [26]. These processes occur at around $-0.4 \mathrm{~V}$ vs $\mathrm{Ag} / \mathrm{AgCl}$, with the peaks referred to as B-B' being related to these faradaic reactions [27].<smiles>Cc1cc2nc3c(=O)[nH]c(=O)nc-3n([13CH3])c3cc(C)c(C)cc3n([13CH2])c2cc1C</smiles>

Scheme 1

However, it has been reported that in neutral and weak basic media the oxidation of flavin-quinone to flavin-hydroquinone goes through the formation of an intermediate (radical semi-quinone), leading to two different overlapped signals [28]. In our case, the redox processes are related to the adsorbed FMN because the peak current increases linearly with the scan rate [25]. The presence of oxidized, semiquinone, and reduced forms of riboflavin has been studied using SERS coupled to electrochemical techniques [29]. Then, the immobilization of FMN on the graphene layers could stabilize the intermediate semiquinone during the oxidation process, with two peaks being clearly observed in the voltammogram. Then, we propose that FMN adsorbed on the graphene surface undergoes two-step reduction, with the overlapped signals $\mathrm{A}^{-\mathrm{A}^{\prime}}$ and B-B' being related to the reduction/oxidation of FMN to flavin-hydroquinone. Finally, the redox processes $\mathrm{C}^{-\mathrm{C}^{\prime}}$ are more difficult to assign and could be related to some impurity belonging to the flavin family (the purity of FMN employed here was between $73 \%$ and $79 \%$ ).

The voltammogram obtained with Pt-Gr-FMN/Au-IDA electrode (Figure 1B) shows the characteristic profile of $\mathrm{Pt}$, the region between $0.05 \mathrm{~V}$ and $0.4 \mathrm{~V}$ corresponds to the so-called adsorption-desorption of hydrogen and anions on the platinum surface, where the redox processes of FMN are also overlapped. The zone between $0.5 \mathrm{~V}$ and $1.2 \mathrm{~V}$ corresponds to the oxidationreduction of the Pt surface. Moreover, an oxidation peak is clearly observed at around $0.65 \mathrm{~V}$ that can be attributed to some oxygen surface groups present in the graphene layers. 

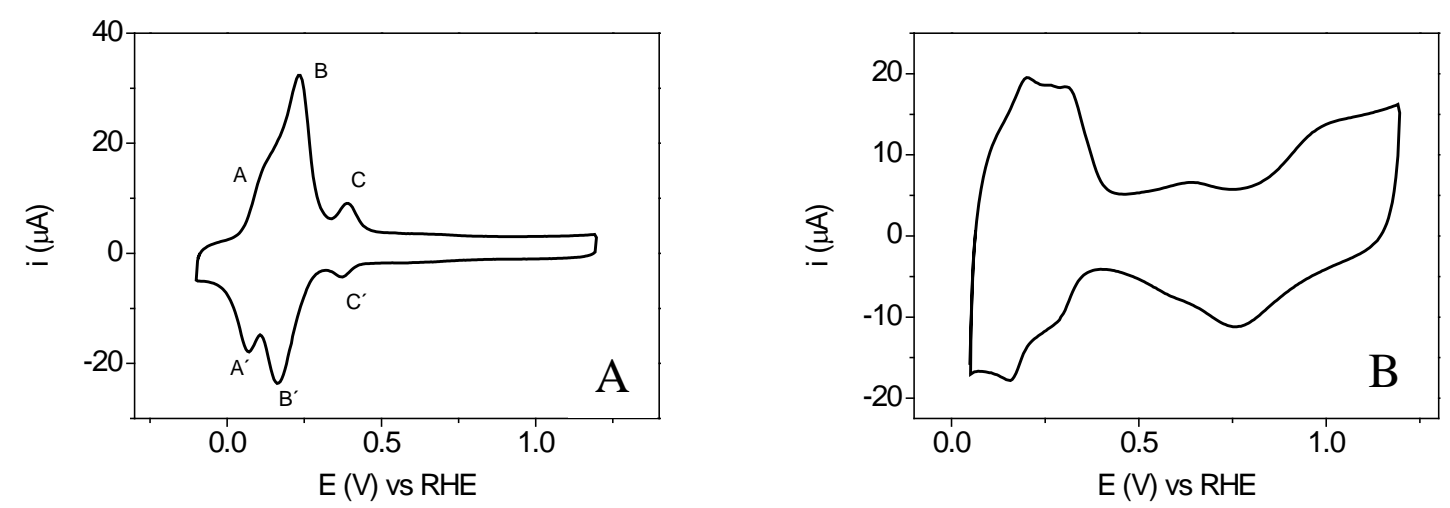

Figure 1. Steady state voltammograms in PBS (0.25 M, pH 7) for: A) Gr-FMN/Au-IDA electrode; B) Pt-Gr-FMN/Au-IDA electrode. $\mathrm{v}_{\text {scan }}=0.1 \mathrm{~V} \mathrm{~s}^{-1}$.

Figures 2-3 show the TEM images of Gr-FMN and Pt-Gr-FMN dispersions, respectively. It can be seen from Figure 2 the expected lamellar nature of the exfoliated graphene sheets. The number of monolayers in each flake obtained with this method (exfoliation and stabilization of graphite using FMN) is between one and five [25].

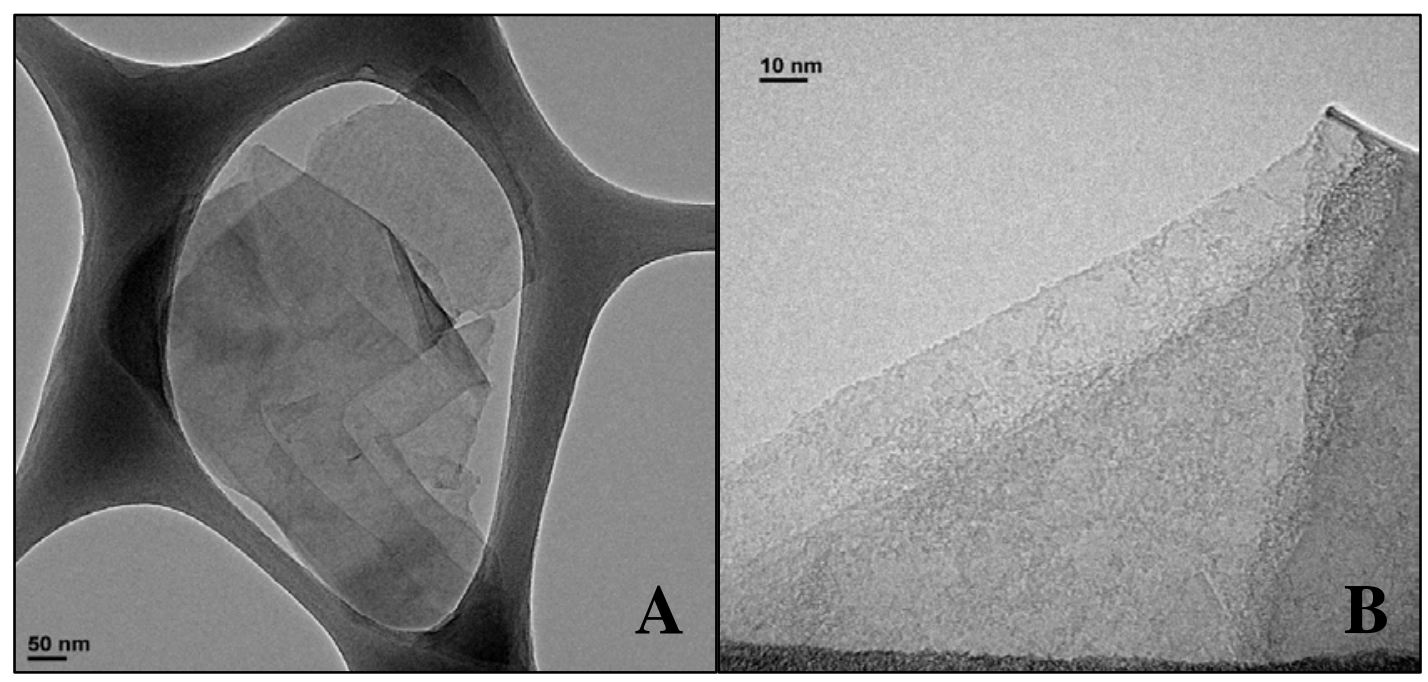

Figure 2. TEM images for Gr-FMN flakes at different magnifications (A-B).

Figure 3 shows a homogeneous distribution of $\mathrm{Pt}$ nanoparticles on the graphene layers, with nanoparticle sizes typically between 3 and $6 \mathrm{~nm}$ (Figure 3b). The amount of Pt on graphene was determined by EDS, resulting in a $(2.52 \pm 0.52) \mathrm{wt} \%$. 


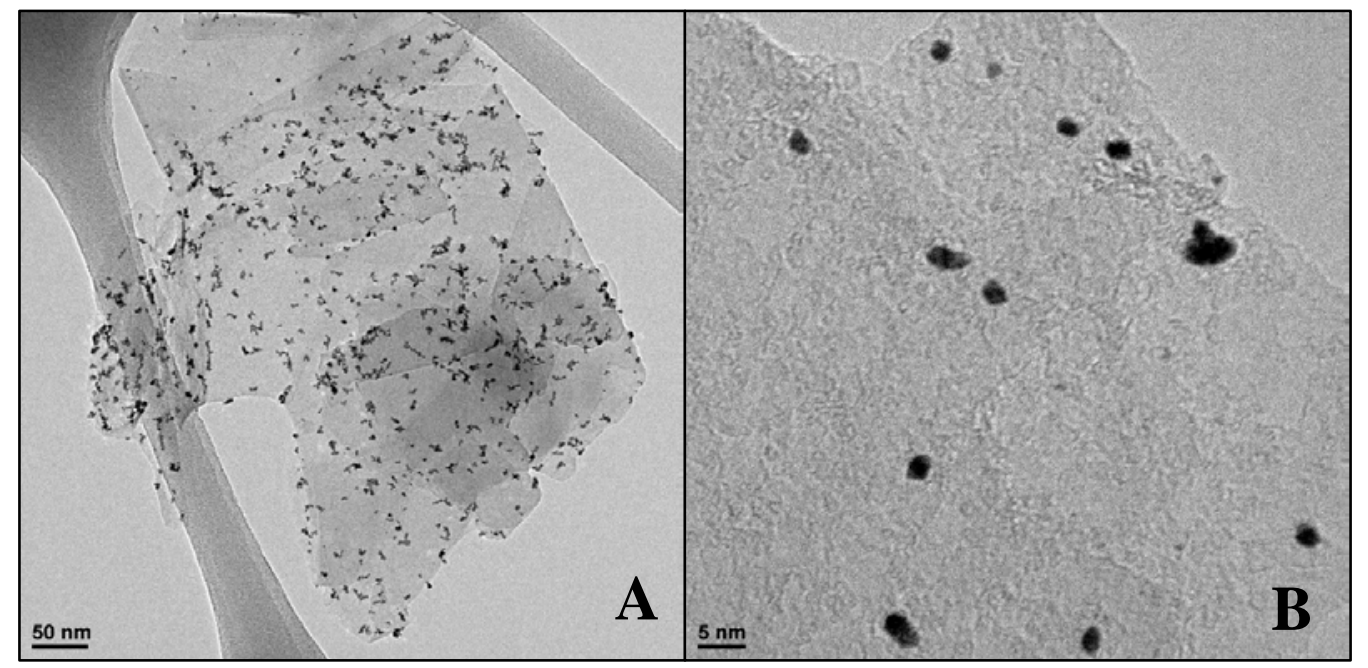

Figure 3. TEM images of Pt-Gr-FMN hybrids at different magnifications (A-B).

\subsection{Electrochemical behavior of UA and AA}

The electrochemical behavior of UA on bare electrode (Au-IDA) and both modified electrodes has been studied by cyclic voltammetry. The Gr-FMN/Au-IDA electrode has been investigated in the potential range in which the redox processes of FMN are not observed. Figure 4 shows the voltammograms obtained during the oxidation of different concentrations of UA on the different electrodes. Figure 4A exhibits the UA oxidation on bare electrode (Au-IDA microelectrode), showing an oxidation current at $1.25 \mathrm{~V}$. Figures 4B-C display the oxidation of UA for Gr-FMN/AuIDA and Pt-Gr-FMN/Au-IDA electrodes. As it can be seen in Figures 4B and 4C, for both electrodes, the oxidation peak related with UA oxidation appears at $0.99 \mathrm{~V}$, providing high oxidation currents than with the bare electrode. According to the results obtained, both modified electrodes with Gr-FMN and Pt-Gr-FMN dispersions show an electrocatalytic effect because the oxidation potential decreases and current increases in comparison to the bare electrode for similar concentrations. In addition, the presence of platinum on graphene also enhances the results obtained with Gr-FMN/Au-IDA (Figure 4B-C) providing higher current intensities than Gr-FMN/Au-IDA electrode. Then, the calibration curves for UA oxidation has been obtained with these two modified electrodes (Figure 4D). All voltammograms have been obtained by triplicate. The oxidation current of UA ( $\mathrm{i}_{\mathrm{UA}}$ ) is linear in the UA concentration range between $60 \mu \mathrm{M}$ and $578 \mu \mathrm{M}$ for Gr-FMN/AuIDA electrode and between $60 \mu \mathrm{M}$ and $345 \mu \mathrm{M}$ for Pt-Gr-FMN/Au-IDA electrode, with LODs of $18 \mu \mathrm{M}$ with both electrodes. The regression models obtained are the following:

Gr-FMN/Au-IDA: $\quad \mathrm{i}_{\mathrm{UA}}(\mu \mathrm{A})=(0.065 \pm 0.003) \mathrm{C}_{\mathrm{UA}}(\mu \mathrm{M})+(1.7 \pm 1.0) ; \mathrm{r}=0.999(\mathrm{~N}=10)$.

Pt-Gr-FMN/Au-IDA: $\quad \mathrm{i}_{\mathrm{UA}}(\mu \mathrm{A})=(0.10 \pm 0.02) \mathrm{C}_{\mathrm{UA}}(\mu \mathrm{M})+(6 \pm 4) ; \mathrm{r}=0.992(\mathrm{~N}=6)$. 
Results obtained by both modified electrodes are outlined in Table 1. It can be observed that the working range for the Pt-Gr-FMN/Au-IDA electrode is shorter than the one obtained in the absence of Pt nanoparticles (Figure 4D, Table 1). This result can be ascribed to the fact that the surface active sites on the Pt nanoparticles are approaching full saturation with UA molecules or the products of their oxidation at the higher UA concentrations tested. The blockage of the platinum surface can be observed in the voltammetric profile, in which the charge of the so-called hydrogen adsorption-desorption zone (between $0 \mathrm{~V}$ and $0.4 \mathrm{~V}$ ) decreases when the UA concentration increases (Figure 4C). When the UA concentration is higher than $345 \mu \mathrm{M}$, it can be considered that all active sites are blocked, and consequently, the oxidation current remains constant with UA concentration.
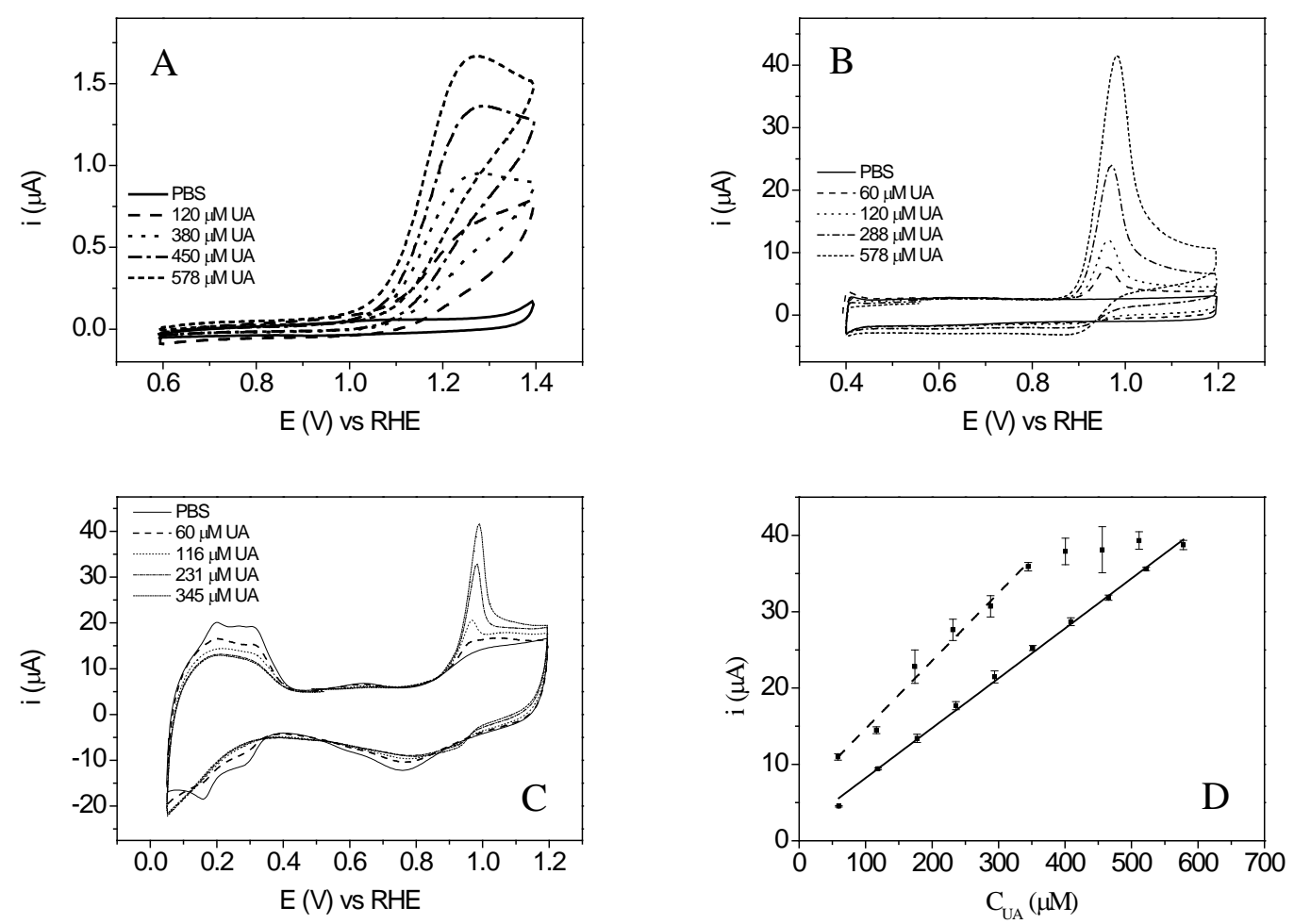

Figure 4. Voltammograms in PBS (0.25 M, pH 7) for successive additions of UA(A-C): A) Bare Au-IDA: solid line: $0 \mu \mathrm{M}$; dashed: $120 \mu \mathrm{M}$; dotted: $380 \mu \mathrm{M}$; dash dotted: $450 \mu \mathrm{M}$; short dashed: $578 \mu \mathrm{M}$; B) Gr-FMN/Au-IDA electrode: solid line: $0 \mu \mathrm{M}$; dashed: $60 \mu \mathrm{M}$; dotted: $120 \mu \mathrm{M}$; dash dotted: $288 \mu \mathrm{M}$; short dashed: $578 \mu \mathrm{M}$. C) Pt/Gr-FMN/Au-IDA electrode: solid line: $0 \mu \mathrm{M}$; dashed:

$60 \mu \mathrm{M}$; dotted: $116 \mu \mathrm{M}$; dash dotted: $231 \mu \mathrm{M}$; short dashed: $345 \mu \mathrm{M}$; $\mathrm{v}_{\text {scan }}=0.1 \mathrm{~V} \cdot \mathrm{s}^{-1}$; D)

Calibration curve for UA quantification in the absence of AA obtained with Gr-FMN/Au-IDA electrode (solid line) and with Pt-Gr-FMNS/Au-IDA electrode (dashed line).

The limit of detection (LOD) was determined empirically, measuring progressively more diluted concentrations of the analyte. The LOD was the lowest concentration whose signal could be clearly distinguished from the blank. Moreover, the limit of quantification was calculated as 3.3 times LOD 
$(\mathrm{LOQ}=3.3 \mathrm{LOD})$. On the other hand, the coefficient of variation values $(\mathrm{CV})$ were obtained for 3 replicates at $177 \mu \mathrm{M}$ concentration level of UA.

In order to identify the voltammetric peaks belonging to AA oxidation, the electrochemical behaviour of bare (Au-IDA) and modified electrodes (Gr-FMN/Au-IDA and Pt-Gr-FMN/Au-IDA) towards the oxidation of AA in the absence of UA was investigated by cyclic voltammetry. Figure 5A shows the voltammograms obtained with a bare Au-IDA electrode in buffer solution and for successive additions of AA to obtain the different concentrations as indicated in the figure. As it can be seen, an anodic signal appears at $0.67 \mathrm{~V}$ belonging to the oxidation of AA. No cathodic signal can be observed in the reverse scan due to the irreversibility of this oxidation. Figure 5B exhibits the voltammograms obtained with the Gr-FMN/Au-IDA in PBS (solid line), AA solution (dashed line) and a mixture of AA and UA (dotted line). Two different oxidation peaks belonging to AA and UA, at potentials around $0.71 \mathrm{~V}$ and $0.99 \mathrm{~V}$ respectively, can be clearly observed. Figure 5C shows the voltammetric behaviour of Pt-Gr-FMN/Au-IDA towards successive additions of AA, which exhibits two anodic peaks at around $0.74 \mathrm{~V}$ and $1.1 \mathrm{~V}$. Then, this second peak could interfere in the determination of UA.

Table 1. Analytical parameters obtained for UA quantification with both modified electrodes (Gr-FMN/Au-IDA and Pt-Gr-FMN/Au-IDA).

\begin{tabular}{ccc}
\hline \multirow{2}{*}{ Parameter } & \multicolumn{2}{c}{ Electrode } \\
\cline { 2 - 3 } & Gr-FMN/Au-IDA & Pt-Gr-FMN/Au-IDA \\
\hline Sensitivity $\left(\mu \mathrm{A} \cdot \mu \mathrm{M}^{-1}\right)$ & $0.065 \pm 0.003$ & $0.10 \pm 0.02$ \\
Intercept $(\mu \mathrm{A})$ & $1.7 \pm 1.0$ & $6 \pm 4$ \\
$\mathrm{r}$ & 0.999 & 0.992 \\
$\mathrm{~N}$ & 10 & 6 \\
Working range $(\mu \mathrm{M})$ & $60-578$ & $60-345$ \\
LOD $(\mu \mathrm{M})$ & 18 & 18 \\
LOQ $(\mu \mathrm{M})$ & 60 & 60 \\
$\mathrm{CV}(\%)$ & 4 & 8 \\
$(\mathrm{n}=3 ; 177 \mu \mathrm{M})$ & &
\end{tabular}

Table 1 exhibits how the presence of Pt nanoparticles provides an enhanced sensitivity as a consequence of the electroactivity of Pt towards UA oxidation; however, the working range decreases in relation to the Gr-FMN/Au-IDA electrode. Linear ranges obtained allow the developed sensor to measure UA levels in serum or urine fluids. 

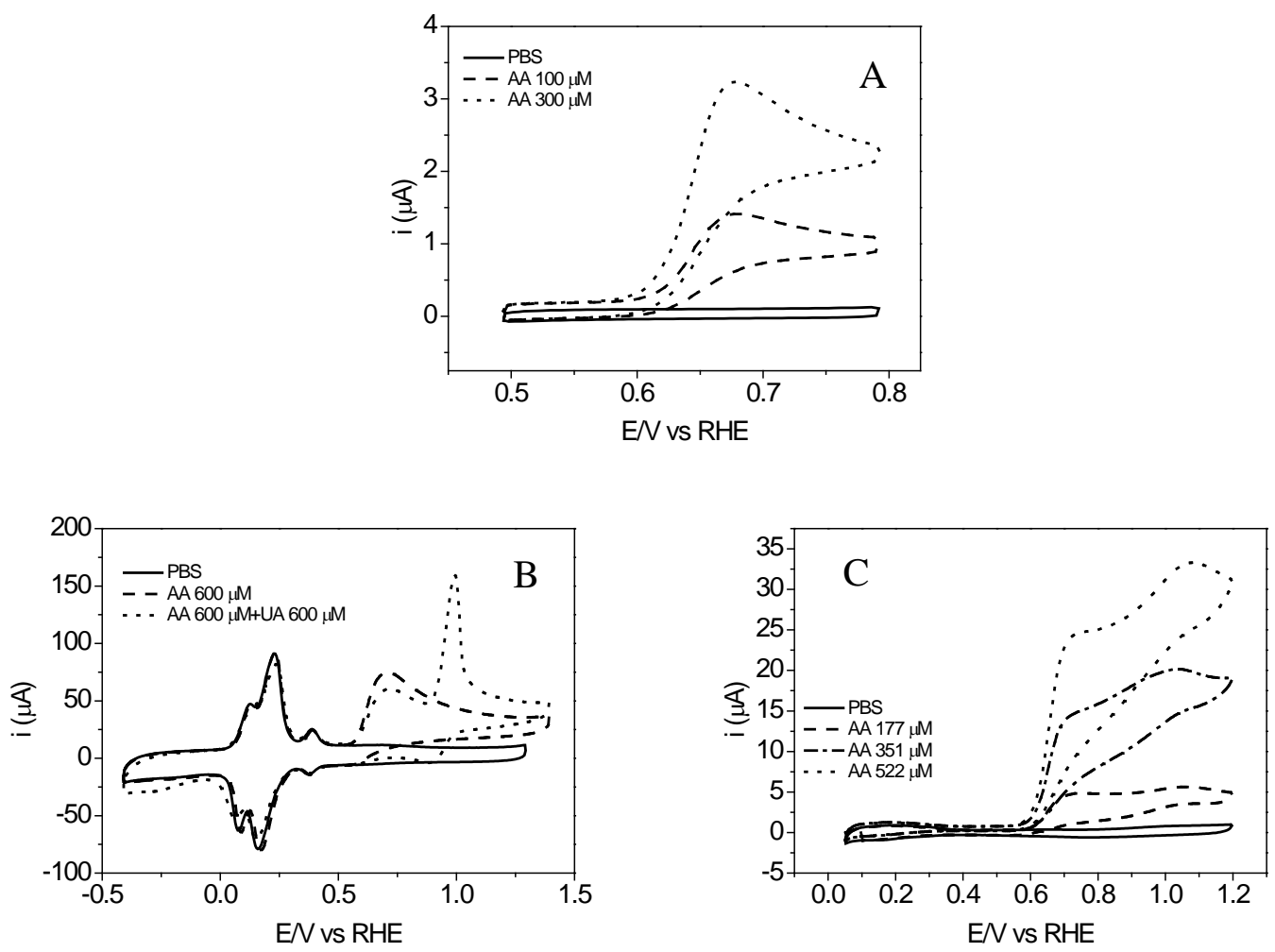

Figure 5. Voltammograms in PBS (0.25 M, pH 7) for successive additions of AA(A-C): A) Bare Au-IDA: solid line: $0 \mu \mathrm{M}$; dashed: $100 \mu \mathrm{M}$; dotted: $300 \mu \mathrm{M}$; B) Gr-FMN/Au-IDA electrode: solid line: $0 \mu \mathrm{M}$; dashed: $600 \mu \mathrm{M}$; dotted: AA $600 \mu \mathrm{M}$ + UA $600 \mu \mathrm{M}$; C) Pt/Gr-FMN/Au-IDA electrode: solid line: $0 \mu \mathrm{M}$; dashed: $177 \mu \mathrm{M}$; dash dotted: $351 \mu \mathrm{M}$; dotted: $522 \mu \mathrm{M}$; $\mathrm{v}_{\text {scan }}=0.1 \mathrm{~V} \cdot \mathrm{s}^{-1}$.

\subsection{Electrochemical determination of UA in the presence of AA}

Possible AA interference was investigated by cyclic voltammetry using a high concentration 3 times higher than the normal levels in serum [30]. The concentration ratios between AA and UA ensure the selectivity of the method towards UA quantification. Figure 6A displays the voltammogram obtained for a bare electrode (Au-IDA) in which two oxidation peaks are clearly observed, the first one corresponding to AA oxidation at $1.07 \mathrm{~V}$ and the second one at $1.28 \mathrm{~V}$ corresponding to UA oxidation. Then, it can be concluded that with this electrode is not possible to separate the oxidation of both compounds. Figures 6B and 6C exhibit the voltammograms obtained for the Gr-FMN/Au-IDA and Pt-Gr-FMN/Au-IDA electrodes respectively at different concentrations of UA in the presence of $250 \mu \mathrm{M}$ AA. Figure 6B shows the behaviour of $\mathrm{Gr}-$ FMN/Au-IDA electrode, the voltammograms show an oxidation peak at $0.74 \mathrm{~V}$ that corresponds to AA oxidation, while the oxidation peak corresponding to UA is maintained at $0.99 \mathrm{~V}$, the same potential than the observed in the absence of AA. Figure 6C exhibits the voltammograms for PtGr-FMN/Au-IDA electrode obtained for successive additions of UA in a $250 \mu \mathrm{M}$ AA solution. It 
is can be observed in all the voltammograms, an oxidation peak corresponding to AA at $0.70 \mathrm{~V}$ and the AA oxidation peak at $0.99 \mathrm{~V}$.

The comparison of the results obtained with modified and non-modified electrodes shows that the modified electrodes show a lower oxidation potentials and higher oxidation currents than the bare electrode for both analytes. Moreover, a larger separation of the oxidation peaks is observed that could improve the resolution of the determination method. The successful separation of the signals related to oxidation of AA and UA was ascribed to the properties of graphene, which offer several advantages for electrochemical sensing applications. Some authors claim that the larger decrease in the oxidation overpotential for AA is probably caused by the interaction between AA and graphene surface, which may accelerate the electron transfer on the modified electrodes. On the other hand, the enhancement in current response may be attributed to the increase of the electrode surface [31]. Moreover, the presence of Pt nanoparticles on the graphene support also produces a slight improvement, showing high currents for lower UA concentrations, due to the high electroactivity of Pt towards several reactions. Moreover, the surface of the electrode contains FMN molecules, which can act as intermediates by accepting electrons from many functional groups and donating electrons to other molecules [26-28]. Then, FMN can act as a mediator of the oxidation of these compounds, thus producing some interactions between the analytes and the surface.

In addition, voltammetric studies for the dependence of the current of AA and UA with the scan rate were performed by using a Pt-Gr-FMN/Au-IDA electrode, indicating that both analytes show the typical behavior of a process where the reactant is in the solution (results not shown).

The calibration curves obtained in the presence of AA are also shown in Figure 6D. It can be observed that the UA oxidation current in the presence of AA $(250 \mu \mathrm{M})$ is linear in the concentration range between $60 \mu \mathrm{M}$ and $578 \mu \mathrm{M}$ with Gr-FMN/Au-IDA and in the range from 60 $\mu \mathrm{M}$ to $288 \mu \mathrm{M}$ for Pt-Gr-FMN/Au-IDA. The following linear relations have been calculated:

Gr-FMN/Au-IDA: $\quad \mathrm{i}_{\mathrm{UA}}(\mu \mathrm{A})=(0.065 \pm 0.003) \cdot \mathrm{C}_{\mathrm{UA}}(\mu \mathrm{M})+(3.0 \pm 1.0) ; \mathrm{r}=0.999(\mathrm{~N}=10)$

Pt-Gr-FMN/Au-IDA: $\quad \mathrm{i}_{\mathrm{UA}}(\mu \mathrm{A})=(0.123 \pm 0.010) \cdot \mathrm{C}_{\mathrm{UA}}(\mu \mathrm{M})-(0 \pm 2) ; \mathrm{r}=0.999(\mathrm{~N}=5)$ 

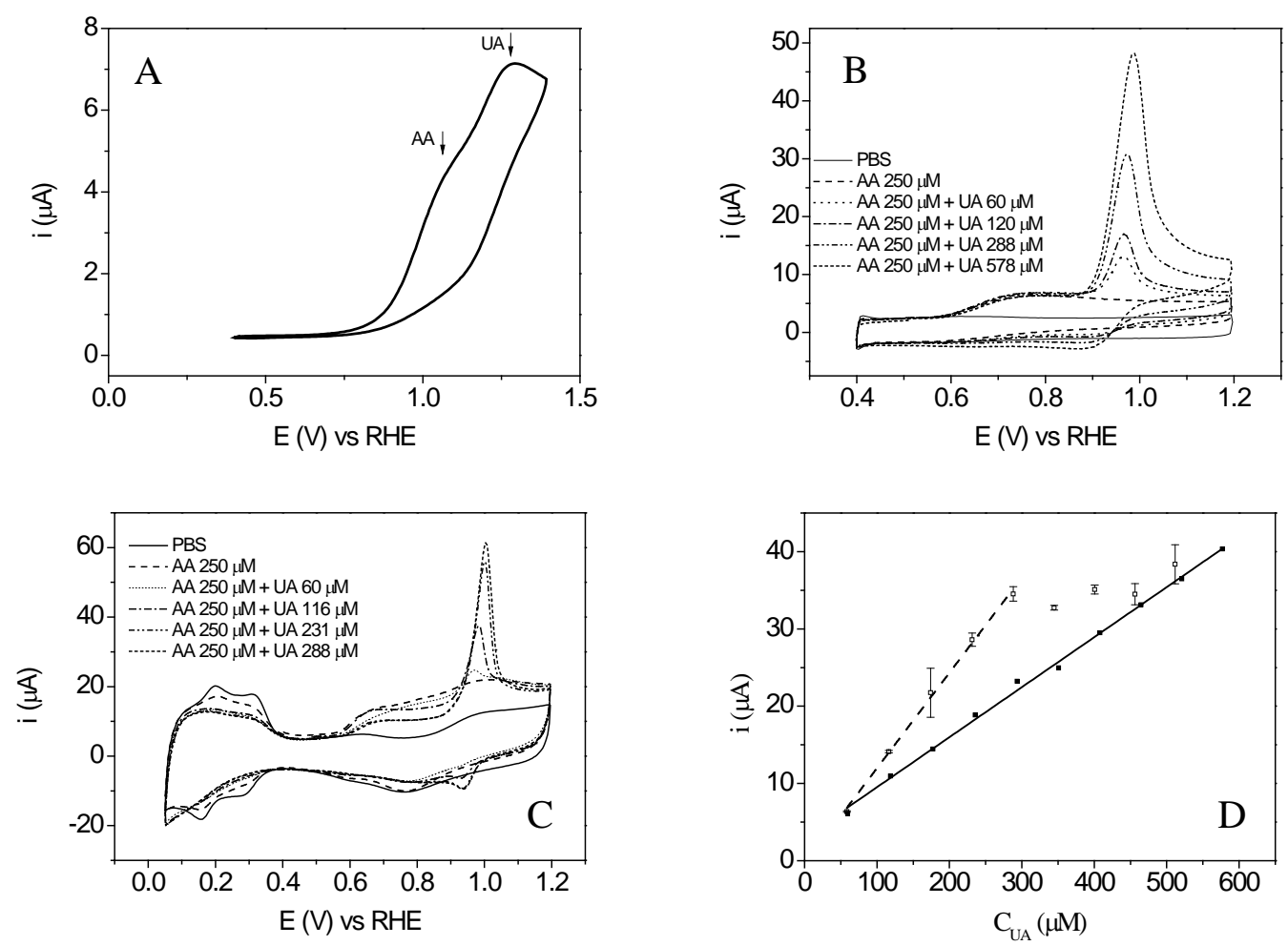

Figure 6. Voltammograms obtained for: A) Bare Au-IDA electrode in 3 mM AA and $87 \mu \mathrm{M}$ UA solutions in PBS; B) Gr-FMN/Au-IDA electrode in $250 \mu \mathrm{M}$ AA solution in PBS for successive additions of UA: solid line: $0 \mu \mathrm{M}$; dashed: $60 \mu \mathrm{M}$; dotted: $120 \mu \mathrm{M}$; dash dotted: $288 \mu \mathrm{M}$; short dashed: $578 \mu \mathrm{M}$.; C) Pt-Gr-FMN/Au-IDA electrode in $250 \mu \mathrm{M}$ AA solution in PBS for successive additions of UA: solid line: $0 \mu \mathrm{M}$; dashed: $60 \mu \mathrm{M}$; dotted: $116 \mu \mathrm{M}$; dash dotted: $231 \mu \mathrm{M}$; short dashed: $288 \mu \mathrm{M} \mathrm{v}_{\text {scan }}=0.1 \mathrm{~V} \cdot \mathrm{s}^{-1}$, D) Calibration curve for UA quantification in the absence of AA obtained with Gr-FMN/Au-IDA electrode (solid line) and with Pt-Gr-FMNS/Au-IDA electrode (dashed line).

Table 2 summarizes the results obtained for Gr-FMN/Au-IDA and Pt-Gr-FMN/Au-IDA electrodes in the presence of AA. The measurements obtained with Pt-Gr-FMN/Au-IDA electrode show slightly higher sensitivity than that of the electrode without Pt nanoparticles. However, its working range is shorter, similar to the case without AA, which is due probably again to saturation of the surface active sites on the metal nanoparticles. Also, the current associated to hydrogen adsorption and desorption (between 0 and $0.4 \mathrm{~V}$ ) decreases when the UA concentration increases. 
Table 2. Analytical parameters obtained for UA quantification in the presence of AA (250 $\mu \mathrm{M})$ with the two modified electrodes (Gr-FMN/Au-IDA and Pt-Gr-FMN/Au-IDA).

\begin{tabular}{ccc}
\hline \multirow{2}{*}{ Parameter } & \multicolumn{2}{c}{ Electrode } \\
\cline { 2 - 3 } & Gr-FMN/Au-IDA & Pt-Gr-FMN/Au-IDA \\
\hline Sensitivity $\left(\mu \mathrm{A} \cdot \mu \mathrm{M}^{-1}\right)$ & $0.065 \pm 0.003$ & $0.123 \pm 0.010$ \\
Intercept $(\mu \mathrm{A})$ & $3.0 \pm 1.0$ & $-0 \pm 2$ \\
$\mathrm{r}$ & 0.999 & 0.999 \\
$\mathrm{~N}$ & 10 & $50-288$ \\
Working range $(\mu \mathrm{M})$ & $60-578$ & 18 \\
LOD $(\mu \mathrm{M})$ & 18 & 60 \\
LOQ $(\mu \mathrm{M})$ & 60 & 14 \\
CV $(\%)$ & 8 & \\
$(\mathrm{n}=3 ; 177 \mu \mathrm{M})$ & & \\
\hline
\end{tabular}

Comparing the results of Table 1 and Table 2, it can be concluded that the working range decreases $\left(\mathrm{C}_{\mathrm{UA}}=288 \mu \mathrm{M}\right)$ when $\mathrm{AA}$ is present in the solution and for the Pt-Gr-FMN/Au-IDA electrode. These results could be as consequence of the blockage of the active sites of platinum surface, which is produced at lower UA concentrations because both AA and UA are adsorbed on the platinum surface. Therefore, for $250 \mu \mathrm{M}$ AA, the intensity of the oxidation peak for UA does not change when the concentration increases from $288 \mu \mathrm{M}$ UA for Pt-Gr-FMN/Au-IDA electrode.

If the values obtained for the sensitivity with and without AA are compared, no significant differences are observed for Gr-FMN/Au-IDA electrode, and only a slightly increase of the slope for Pt-Gr-FMN/Au-IDA electrode $\left(0.10 \pm 0.02 \mu \mathrm{A} \mu \mathrm{M}^{-1}\right.$ (without AA) vs $0.123 \pm 0.010 \mu \mathrm{A} \mu \mathrm{M}^{-}$ ${ }^{1}$ (with AA)) is obtained.

Finally, in order to assess whether AA interferes in the UA measurements, that is, whether the results obtained in the presence of AA in the solution are the same as without AA, a statistical test was applied. It involves plotting the results of the measurement of UA without AA in one of the axis (Y axis), and the results obtained with AA in the other axis (X-axis), for the same electrode. If the results obtained with and without AA are the same, a straight line with a slope amounting to one and crossing the origin (intercept zero) will be obtained [32].

Figure 7 shows the plots obtained from the results for the UA oxidation with and without AA for the Gr-FMN/Au-IDA and Pt-Gr-FMN/Au-IDA electrodes. The plot obtained for Gr-FMN/Au-IDA 
electrode (Figure 7A) shows a very good correlation between both results in the presence and absence of AA. The linear equation obtained is: $i_{U A}(\mu A)=(0.99 \pm 0.04) \cdot i_{U A}+A A(\mu A)-(0.8 \pm 0.9)$ with a correlation coefficient of 0.999 . Then, it can be concluded that AA does not interfere in the UA quantification using the Gr-FMN/Au-IDA electrode.
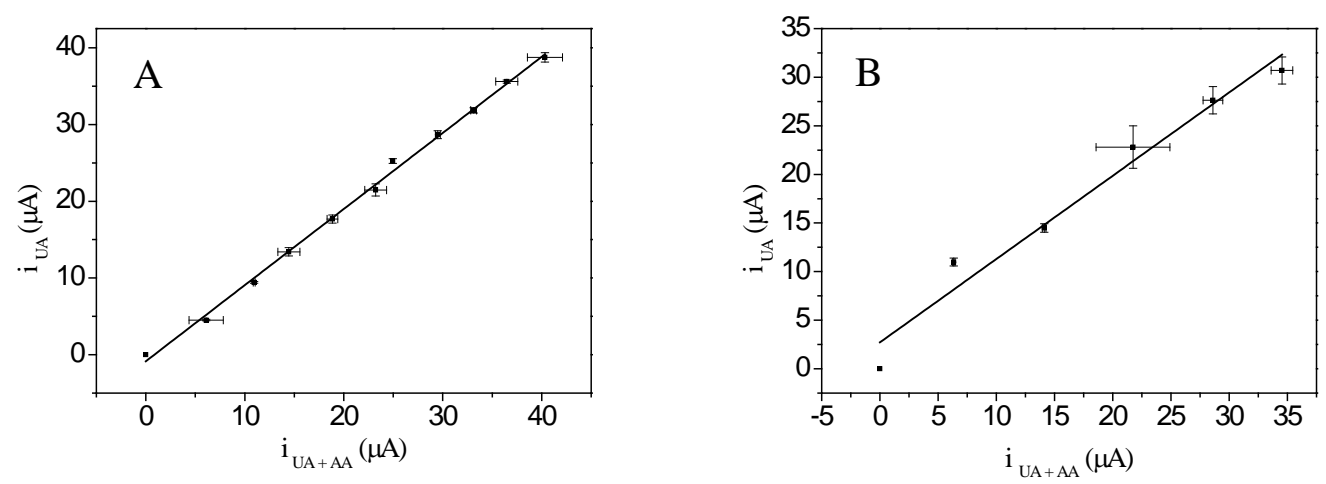

Figure 7. Regression lines of $i_{\mathrm{UA}}$ vs $\mathrm{i}_{\mathrm{AA}+\mathrm{UA}}$ : A) Gr-FMN/Au-IDA electrode;

B) Pt-Gr-FMN/Au-IDA electrode. Error bars are represented by the standard deviation obtained with three replicates.

Figure 7B shows the correlation obtained for Pt-Gr-FMN/Au-IDA electrode with and without AA. The linear relationship obtained is $\mathrm{i}_{\mathrm{UA}}(\mu \mathrm{A})=(0.8 \pm 0.2) \cdot \mathrm{i}_{\mathrm{UA}+\mathrm{AA}}(\mu \mathrm{A})+(4 \pm 4)$ and a correlation coefficient of 0.984 . In this case, the correlation is lower than with the other electrode, but it can be concluded that AA does not significantly interfere in the UA quantification using the Pt-GrFMN/Au-IDA electrode. However, if the results obtained for Gr-FMN/Au-IDA and Pt-GrFMN/Au-IDA electrodes are compared, it can be noticed several differences. The correlation coefficient is much higher for the Gr-FMN/Au-IDA electrode, indicating that the results obtained with and without AA are more similar with this electrode. However, the sensitivity obtained with Pt-Gr-FMN/Au-IDA electrode is higher than the value obtained without platinum, then, this Pt-GrFMN/Au-IDA electrode provides the most auspicious results that can be ascribed to the synergistic effect between the high conductivity and large surface area of graphene and the high catalytic activity of Pt nanoparticles.

The lifetime of the electrodes is another important parameter for practical application. The Gr-FMN and Pt-Gr-FMN dispersions are stable for at least six months, without showing significant precipitation [25]. After this time, the FMN could degrade in the aqueous graphene dispersion due to its photosensitivity. Then, it can be considered that the lifetime of the electrodes could be around six months. 
Finally, Table 3 shows a comparison of some analytical parameters obtained in this work, with others obtained from the literature. It can be observed that the proposed material electrode is competitive, representing therefore a good and interesting alternative for sensor applications. In addition, no sensors based in cyclic voltammetry detection with sensitivity higher than the one reached in the present work were found in literature.

Table 3. Comparison of analytical parameters for several electrochemical UA sensors by voltammetric detection.

\begin{tabular}{|c|c|c|c|c|c|}
\hline Parameter & Method & $\begin{array}{l}\text { Sensitivity } \\
\left(\mu \mathrm{A} \cdot \mu \mathrm{M}^{-1}\right)\end{array}$ & $\begin{array}{l}\mathrm{LOD} \\
(\mu \mathrm{M})\end{array}$ & $\begin{array}{c}\text { Linear } \\
\text { Range } \\
(\mu \mathrm{M})\end{array}$ & Ref. \\
\hline MBI-Au & $\begin{array}{c}\text { Cyclic } \\
\text { Voltammetry }\end{array}$ & $\begin{array}{c}0.0152 \pm \\
0.0005\end{array}$ & 1 & $1-300$ & [3] \\
\hline Bare CPE & DPV & 0.0238 & 5.63 & $0-100$ & {$[10]$} \\
\hline Ag-PPy/GCE & DPV & 0.1818 & 0.5 & $2-100$ & {$[21]$} \\
\hline $\begin{array}{c}\text { GCE- } \\
\text { CoPc/MWCNT }\end{array}$ & $\begin{array}{c}\text { Cyclic } \\
\text { Voltammetry }\end{array}$ & 0.02 & 260 & $260-4000$ & {$[22]$} \\
\hline $\begin{array}{c}\text { AuNPs- } \\
\text { MWCNTs/GC }\end{array}$ & DPV & 0.037 & 2 & $4-300$ & [23] \\
\hline $\mathrm{MoS}_{2} / \mathrm{rGO}$ & DPV & 0.141 & 0.46 & $1.38-745$ & [33] \\
\hline $\mathrm{AuNPs}_{(\mathrm{EDAS})}{ }^{-\mathrm{rGO}}$ & $\begin{array}{c}\text { Cyclic } \\
\text { Voltammetry }\end{array}$ & 0.008 & 0.5 & $0.5-50$ & [34] \\
\hline $\begin{array}{c}\text { Pt-Gr-FMN/Au- } \\
\text { IDA }\end{array}$ & $\begin{array}{c}\text { Cyclic } \\
\text { Voltammetry }\end{array}$ & $0.123 \pm 0.010$ & 18 & $60-284$ & $\begin{array}{l}\text { This } \\
\text { work }\end{array}$ \\
\hline Gr-FMN/Au-IDA & $\begin{array}{c}\text { Cyclic } \\
\text { Voltammetry }\end{array}$ & $0.065 \pm 0.003$ & 18 & $60-578$ & $\begin{array}{l}\text { This } \\
\text { work }\end{array}$ \\
\hline
\end{tabular}

\section{Conclusions}

Graphene flakes exfoliated using flavin mononucleotide and platinum nanoparticles supported on graphene flakes have been electrochemical characterized by cyclic voltammetry. The dispersions of these materials have been used in the modification of gold interdigitated microelectrodes. An electrochemical method has been developed for the quantification of uric acid in the presence of ascorbic acid by cyclic voltammetry. The analyses have been carried out in phosphate buffer solution to simulate physiological human fluids (PBS, $0.25 \mathrm{M}, \mathrm{pH}$ 7). Two different modified electrodes have been studied: Gr-FMN/Au-IDA and Pt-Gr-FMN/Au-IDA, with the Pt-Gr-FMN/AuIDA electrode. The Gr-FMN/Au-IDA electrode exhibits a working range of the UA oxidation 
current with the UA concentration being between $60 \mu \mathrm{M}$ and $578 \mu \mathrm{M}$, achieving a LOD of $18 \mu \mathrm{M}$. When AA is in the UA solution, these values remain constant for this electrode. For the Pt-GrFMN/Au-IDA electrode, the working range obtained for the UA oxidation current with the concentration is not as good as with the Gr-FMN/Au-IDA electrode due to the adsorption of UA and AA on the Pt surface, resulting in a shorter working range (between $60 \mu \mathrm{M}$ and $288 \mu \mathrm{M}$ ). Even though the Gr-FMN/Au-IDA electrode offers a larger linear range, the presence of Pt nanoparticles has its own advantages, including a higher sensitivity $\left(0.123 \pm 0.010 \mu \mathrm{A} \mu \mathrm{M}^{-1}\right)$ that is approximately twice the sensitivity value of the Gr-FMN/Au-IDA electrode. As a consequence of the analytical figures of merit obtained in this work, the developed graphene based-materials becomes an alternative to develop electrochemical sensors, able to measure UA levels in some physiological fluids.

\section{Acknowledgement}

This work is supported by the Generalitat Valenciana (Prometeo2013/038) and by the Spanish Ministerio de Economía y Competitividad (MAT2013-42007-P). We also acknowledge partial funding of this work by Ministerio de Economía y Competitividad and the European Regional Development Fund (ERDF) through project MAT2015-69844-R as well as by Plan de Ciencia, Tecnología e Innovación 2013-2017 del Principado de Asturias and ERDF (project GRUPIN14056). A. Abellán-Llobregat also thanks the Generalitat Valenciana for her fellowship and M. AyánVarela acknowledges his predoctoral contract (FPI) from Ministerio de Economía y Competitividad.

\section{References}

[1] M.K. Kutzing, B.L. Firestein, Altered uric acid levels and disease states, J. Pharmacol. Exp. Ther. 324 (2008) 1-7. doi:10.1124/jpet.107.129031.

[2] D. Lakshmi, M.J. Whitcombe, F. Davis, P.S. Sharma, B.B. Prasad, Electrochemical detection of uric acid in mixed and clinical samples: a review, Electroanalysis. 23 (2011) 305-320. doi:10.1002/elan.201000525.

[3] C. Retna Raj, T. Ohsaka, Voltammetric detection of uric acid in the presence of ascorbic acid at a gold electrode modified with a self-assembled monolayer of heteroaromatic thiol, J. Electroanal. Chem. 540 (2003) 69-77. doi:10.1016/S0022-0728(02)01285-8.

[4] T.R. Merriman, H.K. Choi, N. Dalbeth, The genetic basis of gout, Rheum. Dis. Clin. N. Am. 40 (2014) 279-290. doi:10.1016/j.rdc.2014.01.009.

[5] Q. Lv, X.-F. Meng, F.-F. He, S. Chen, H. Su, J. Xiong, P. Gao, X.-J. Tian, J.-S. Liu, Z.-H. Zhu, K. Huang, C. Zhang, High serum uric acid and increased risk of type 2 diabetes: a systemic review and meta-analysis of prospective cohort studies, PLOS ONE. 8 (2013) e56864. doi:10.1371/journal.pone.0056864. 
[6] A. So, B. Thorens, Uric acid transport and disease, J. Clin. Invest. 120 (2010) 1791-1799. doi:10.1172/JCI42344.

[7] M. Alderman, K.J.V. Aiyer, Uric acid: role in cardiovascular disease and effects of losartan, Curr. Med. Res. Opin. 20 (2004) 369-379. doi:10.1185/030079904125002982.

[8] D. Remane, S. Grunwald, H. Hoeke, A. Mueller, S. Roeder, M. von Bergen, D.K. Wissenbach, Validation of a multi-analyte HPLC-DAD method for determination of uric acid, creatinine, homovanillic acid, niacinamide, hippuric acid, indole-3-acetic acid and 2-methylhippuric acid in human urine, J. Chromatogr. B. 998-999 (2015) 40-44. doi:10.1016/j.jchromb.2015.06.021.

[9] A.T. Lawal, Synthesis and utilisation of graphene for fabrication of electrochemical sensors, Talanta. 131 (2015) 424-443. doi:10.1016/j.talanta.2014.07.019.

[10] E. Colín-Orozco, S. Corona-Avendaño, M.T. Ramírez-Silva, M. Romero-Romo, M. PalomarPardavé, On the electrochemical oxidation of dopamine, ascorbic acid and uric acid onto a bare carbon paste electrode from a $0.1 \mathrm{M} \mathrm{NaCl}$ aqueous solution at pH 7, Int. J. Electrochem. Sci. 7 (2012) 6097-6105.

[11] T. Yao, Y. Taniguchi, T. Wasa, S. Musha, Anodic voltammetry and its analytical application to the detection and simultaneous determination of hypoxanthine, xanthine, and uric acid, Bull. Chem. Soc. Jpn. 51 (1978) 2937-2941. doi:10.1246/bcsj.51.2937.

[12] S.A. John, Simultaneous determination of uric acid and ascorbic acid using glassy carbon electrodes in acetate buffer solution, J. Electroanal. Chem. 579 (2005) 249-256. doi:10.1016/j.jelechem.2005.02.012.

[13] L. Zhang, X. Jiang, Attachment of gold nanoparticles to glassy carbon electrode and its application for the voltammetric resolution of ascorbic acid and dopamine, J. Electroanal. Chem. 583 (2005) 292-299. doi:10.1016/j.jelechem.2005.06.014.

[14] J. Huang, Y. Liu, H. Hou, T. You, Simultaneous electrochemical determination of dopamine, uric acid and ascorbic acid using palladium nanoparticle-loaded carbon nanofibers modified electrode, Biosens. Bioelectron. 24 (2008) 632-637. doi:10.1016/j.bios.2008.06.011.

[15] S. Wei, F. Zhao, B. Zeng, Electrochemical behavior and determination of uric acid at singlewalled carbon nanotube modified gold electrodes, Microchim. Acta. 150 (2005) 219-224. doi:10.1007/s00604-005-0368-X.

[16] S. Qi, B. Zhao, H. Tang, X. Jiang, Determination of ascorbic acid, dopamine, and uric acid by a novel electrochemical sensor based on pristine graphene, Electrochimica Acta. 161 (2015) 395-402. doi:10.1016/j.electacta.2015.02.116.

[17] M. Pumera, Electrochemistry of graphene: new horizons for sensing and energy storage, Chem. Rec. 9 (2009) 211-223. doi:10.1002/tcr.200900008.

[18] M. Pumera, Graphene-based nanomaterials and their electrochemistry, Chem. Soc. Rev. 39 (2010) 4146-4157. doi:10.1039/C002690P.

[19] N.G. Shang, P. Papakonstantinou, M. McMullan, M. Chu, A. Stamboulis, A. Potenza, S.S. Dhesi, H. Marchetto, Catalyst-free efficient growth, orientation and biosensing properties of multilayer graphene nanoflake films with sharp edge planes, Adv. Funct. Mater. 18 (2008) 3506-3514. doi:10.1002/adfm.200800951.

[20] Z. Sun, H. Fu, L. Deng, J. Wang, Redox-active thionine-graphene oxide hybrid nanosheet: One-pot, rapid synthesis, and application as a sensing platform for uric acid, Anal. Chim. Acta. 761 (2013) 84-91. doi:10.1016/j.aca.2012.11.057.

[21] K. Ghanbari, N. Hajheidari, Simultaneous electrochemical determination of dopamine, uric acid and ascorbic acid using silver nanoparticles deposited on polypyrrole nanofibers, J. Polym. Res. 22 (2015) 1-9. doi:10.1007/s10965-015-0797-0.

[22] J. de Fátima Giarola, A. César Pereira, Development and application of a sensor based on carbonaceous materials and cobalt phthalocyanine composite for electrochemical determination of uric acid, Electroanalysis. 28 (2016) 1348-1355. doi:10.1002/elan.201501032.

[23] Y. Pan, Y. Li, J. Jia, H. Chen, Selective determination of uric acid in the presence of ascorbic acid using layer-by-layer gold nanoparticles, tin oxide nanoparticles and multi-walled carbon 
nanotubes assembled multilayer films, Electrochemistry. 83 (2015) 956-961.

doi:10.5796/electrochemistry.83.956.

[24] H. Zhang, X. Bo, L. Guo, Electrochemical preparation of Pt nanoparticles supported on porous graphene with ionic liquids: Electrocatalyst for both methanol oxidation and $\mathrm{H} 2 \mathrm{O} 2$ reduction, Electrochimica Acta. 201 (2016) 117-124. doi:10.1016/j.electacta.2016.03.102.

[25] M. Ayán-Varela, J.I. Paredes, L. Guardia, S. Villar-Rodil, J.M. Munuera, M. Díaz-González, C. Fernández-Sánchez, A. Martínez-Alonso, J.M.D. Tascón, Achieving extremely concentrated aqueous dispersions of graphene flakes and catalytically efficient graphene-metal nanoparticle hybrids with flavin mononucleotide as a high-performance stabilizer, ACS Appl. Mater. Interfaces. 7 (2015) 10293-10307. doi:10.1021/acsami.5b00910.

[26] S.L.J. Tan, R.D. Webster, Electrochemically induced chemically reversible proton-coupled electron transfer reactions of riboflavin (vitamin B2), J. Am. Chem. Soc. 134 (2012) 59545964. doi:10.1021/ja300191u.

[27] M. Roushani, Z. Abdi, Novel electrochemical sensor based on graphene quantum dots/riboflavin nanocomposite for the detection of persulfate, Sens. Actuators B Chem. 201 (2014) 503-510. doi:10.1016/j.snb.2014.05.054.

[28] O.S. Ksenzhek, S.A. Petrova, Electrochemical properties of flavins in aqueous solutions, Bioelectrochem. Bioenerg. 11 (1983) 105-127. doi:10.1016/0302-4598(83)80086-5.

[29] M.R. Bailey, Z.D. Schultz, SERS speciation of the electrochemical oxidation-reduction of riboflavin, Analyst. 141 (2016) 5078-5087. doi:10.1039/C6AN01054G.

[30] A.A. Fowler, A.A. Syed, S. Knowlson, R. Sculthorpe, D. Farthing, C. DeWilde, C.A. Farthing, T.L. Larus, E. Martin, D.F. Brophy, S. Gupta, Medical Respiratory Intensive Care Unit Nursing, B.J. Fisher, R. Natarajan, Phase I safety trial of intravenous ascorbic acid in patients with severe sepsis, J. Transl. Med. 12 (2014) 32. doi:10.1186/1479-5876-12-32.

[31] D.-F. Han, C.-S. Shan, L.-P. Guo, L. Niu, D.-X. Han, Electro-oxidation of ascorbic acid on PVP-stabilized graphene electrode, Chem. Res. Chin. Univ. 26 (2010) 287-290.

[32] Miller, J., Miller, J.C., Statistics and chemometrics for analytical chemistry, 6th edition, Pearson, 6th edition, 2010. http://197.14.51.10:81/pmb/CHIMIE/0273730428.pdf (accessed March 9, 2016).

[33] L. Xing, Z. Ma, A glassy carbon electrode modified with a nanocomposite consisting of MoS2, Microchim. Acta. 183 (2015) 257-263. doi:10.1007/s00604-015-1648-8.

[34] V. Vinoth, J.J. Wu, S. Anandan, Sensitive electrochemical determination of dopamine and uric acid using AuNPs(EDAS)-rGO nanocomposites, Anal. Methods. 8 (2016) 4379-4390. doi:10.1039/C6AY00335D. 